\title{
Article \\ Comparative Transcriptome Analysis Reveals Sex-Biased Expression of Hormone-Related Genes at an Early Stage of Sex Differentiation in Red Bayberry (Morella rubra)
}

\author{
Huimin Jia ${ }^{1,2, *}$, Lan Zhao ${ }^{2}$, Yan Wang ${ }^{2}$, Hongxia $W^{3}{ }^{\mathbb{D}}$, Haibo Zhao ${ }^{2}$, Yifan Zhu ${ }^{2}$, Yun Jiao ${ }^{4} \mathbb{D}$, Guoyun Wang ${ }^{5}$, \\ Chaochao Zhou ${ }^{5}$, Chunhui Huang ${ }^{1}$, Huijuan Jia ${ }^{2}$ and Zhongshan Gao ${ }^{2, *}$ \\ 1 College of Agronomy, Jiangxi Agricultural University, Nanchang 330045, China; lindahch@126.com \\ 2 Fruit Science Institute, College of Agriculture and Biotechnology, Zhejiang University, \\ Hangzhou 310058, China; 21716040@zju.edu.cn (L.Z.); 11816010@zju.edu.cn (Y.W.); \\ zhaohaibo@zju.edu.cn (H.Z.); 21916154@zju.edu.cn (Y.Z.); jiahuijuan@zju.edu.cn (H.J.) \\ 3 Key Laboratory of Tropical Fruit Biology, Ministry of Agriculture, South Subtropical Crops Research Institute, \\ Chinese Academy of Tropical Agricultural Sciences, Zhanjiang 524091, China; whx1106@163.com \\ 4 Institute of Forestry, Ningbo Academy of Agricultural Science, Ningbo 315400, China; jydyx@163.com \\ 5 Yuyao Agricultural Technology Extension Service Station, Ningbo 315400, China; \\ yywgy701218@163.com (G.W.); zhoucc1988@163.com (C.Z.) \\ * Correspondence: jiahuimin1988@163.com (H.J.); gaozhongshan@zju.edu.cn (Z.G.)
}

\section{check for}

updates

Citation: Jia, H.; Zhao, L.; Wang, Y.; Wu, H.; Zhao, H.; Zhu, Y.; Jiao, Y.; Wang, G.; Zhou, C.; Huang, C.; et al. Comparative Transcriptome Analysis Reveals Sex-Biased Expression of Hormone-Related Genes at an Early Stage of Sex Differentiation in Red Bayberry (Morella rubra). Horticulturae 2022, 8, 183. https://doi.org/ $10.3390 /$ horticulturae 8020183

Academic Editor: Dilip R. Panthee

Received: 5 January 2022

Accepted: 15 February 2022

Published: 21 February 2022

Publisher's Note: MDPI stays neutral with regard to jurisdictional claims in published maps and institutional affiliations.

Copyright: (C) 2022 by the authors. Licensee MDPI, Basel, Switzerland. This article is an open access article distributed under the terms and conditions of the Creative Commons Attribution (CC BY) license (https:// creativecommons.org/licenses/by/ $4.0 /)$.

\begin{abstract}
The molecular mechanism of sex development and differentiation in the economically important dioecious fruit tree, red bayberry (Morella rubra), was revealed using next-generation transcriptome sequencing (NGS), and comparative analyses were used to identify differentially expressed genes (DEGs) in female and male flower buds. A total of 7029 of these DEGs were identified at two early development stages. KEGG pathway enrichment analysis revealed that plant hormone signal transduction was significantly overrepresented, and 91 genes related to hormones were identified. An analysis of 7029 DEGs revealed 161 hormone-related genes, with the 42 related to auxin and 26 related to ethylene being the most highly represented. A total of 62 genes were significantly up-regulated in females and 29 were in males, with 18 of them specifically expressed in females and 10 in males. A total of 415 transcription factors were identified, with 129 genes up-regulated in females and 53 in males. Moreover, 38 had female-specific expression and 18 had male-specific expression. Using weighted gene co-expression network analysis (WGCNA), two modules were found to be associated with sexual type. In the module coded light-green, there were five genes related to hormones, one to flower development and ten transcription factors with four genes specifically expressed in the males and four in females. The hub gene in the light-green module is MROTCONS_00017483.1 (ACO), which is involved in ethylene biosynthesis and had male-specific expression. Among the transcription factors, three of the four male-specific expressed genes involved in flavonoid biosynthesis, including the MYB gene MR1TCONS_00020658.1 and two BHLH genes, MR6G001563.1 and MR8G020751.1, played important roles in male floral differentiation. In the darkcyan module, six hormone-related genes, five transcription factors and three flower development genes were identified with the hub gene MR1G019545.1 (ETR1), which participates in the ethylene signaling pathway, and $M R 4 G 023618.1$, which encodes the $\mathrm{C} 3 \mathrm{H}$ zinc finger transcription factor. These results indicate that ethylene is the key hormone that interacts with other hormones and transcription factors to regulate sex differentiation in the red bayberry, which also provides new insights into the mechanism of sex determination and differentiation in the red bayberry.
\end{abstract}

Keywords: Morella rubra; sex determination and differentiation; hormones; ethylene; transcription factor

\section{Introduction}

The evolution of sex differentiation is an interesting topic because it is important to strategies for outbreeding, the prevention of inbreeding depression and a vital source 
of genetic variation. About $10 \%$ of flowering plants have separate unisexual flowers in different individuals (dioecy) or in the same individual (monoecy) [1]. For the mechanisms of unisexual flower development, two categories of unisexual flowers have been defined [2,3]. In type I, bisexual flowers become unisexual through the termination of the development of the androecium or gynoecium. The sex differentiation occurs at the particular developmental stage when one of the reproductive organs is arrested. Flowers of type II are unisexual from inception, and sex differentiation occurs before the formation of female or male organ primordia [3].

Sex determination has evolved multiple times, independently, suggesting various genetic mechanisms of unisexual flower formation are at play in different species [3,4]. The genetic mechanism of sex determination in dioecy is often thought to be controlled by a putative sex chromosome. This includes a dominant XY system (male heterogamety), such as the one found in diploid persimmon [5] and papaya [6], and, in a few cases, a ZW system (female heterogamety), as found in the red bayberry [7] and wild species of the allooctoploid strawberry [8]. Sex-determining genes are located in the non-recombining region of the $\mathrm{Y}$ or $\mathrm{W}$ chromosome. For example, in garden asparagus $[9,10]$ and kiwifruit $[11,12]$, the two sex-determining genes are in the sex determination region (SDR), consistent with the classical 'two-mutation model' for the evolution of dioecy from hermaphroditism via gynodioecy.

Hormones and transcription factors are coded with genetic factors, and can also regulate sex differentiation $[13,14]$. Several sex-differentiating genes have been found to be involved in hormone biosynthesis or signaling pathways. In kiwifruit, a cytokinin response regulator Shy Girl (SyGI) has been identified as a sex-determining gene that acts as the suppressor of feminization [11]. In the cucumber, four of five genes controlling flower sex type encode the key enzymes involved in ethylene biosynthesis, including CsACS1G (F) [15], CsACS2 (M) [16], CsACS11 (A) [17] and one CsACO2 [18]. In addition, the zinc finger transcription factor WIP1 suppresses the female flower to control unisexual flower development in the cucumber and melon [18].

Morella rubra, commonly known as the red bayberry, is the only cultivated edible fruit species in the Myricaceae family, and it is widely distributed in south China [19,20]. Most species in Myricaceae are dioecious, with very few monoecious individuals [21,22], and with male, female and monoecious flowers on different plants. It is difficult to identify sex before flowering, as there is no obvious difference between males and females. The sex type of the red bayberry is controlled by the sex chromosomes: ZW for females, $\mathrm{ZZ}$ for males. A $59 \mathrm{~kb}$ non-recombining region in the $\mathrm{W}$ chromosomes harboring seven candidate genes has been identified [7]. The red bayberry flower is a type II unisexual flower, in which only female or male organ primordia are initiated. The male flowers are compound catkins, which are surrounded by bracts without receptacles or pedicels, and each of them has two stamens (Figure 1a). The female flowers are catkins (Figure 1b), and the ovary is unilocular with a Y-shaped stigma [20]. A time course experiment indicated that the critical period for flower bud development is from July to September. The red bayberry is also a good species for sex determination mechanistic studies because of its small genome size $(320 \mathrm{Mb}), \mathrm{ZW}$ chromosome and viable WW genotype (super female) individuals [7,23]. Although seven female-specific gene and molecular markers linked to the sex type have been identified [7], the molecular mechanism and the associated and regulated genes that govern sex determination are not well understood in the red bayberry.

With the continuous progress observed in high-throughput sequencing, transcriptome analyses have been reported in many species to unravel the molecular process regulating the determination of unisexual flowers, such as in papaya [24], spinach [25,26] and Jatropha curcas L. [27]. Although a comparative analysis of gene expression between female and the monoecious mutant red bayberry flowers has been reported [28], the flower samples used were taken during late stages (December) of flower development, when sex differentiation is completed and the key genes regulating sex determination might be no longer expressed. In the present study, transcriptomes analyses of red bayberry floral 
buds from female and male trees were performed, before the initiation of reproductive organ primordia, aiming to capture useful information to understand the mechanism of sex differentiation and floral development in the red bayberry. The results of this study can also be used to guide cross-breeding between two cultivars by manually inducing male flowers in female plants.

a

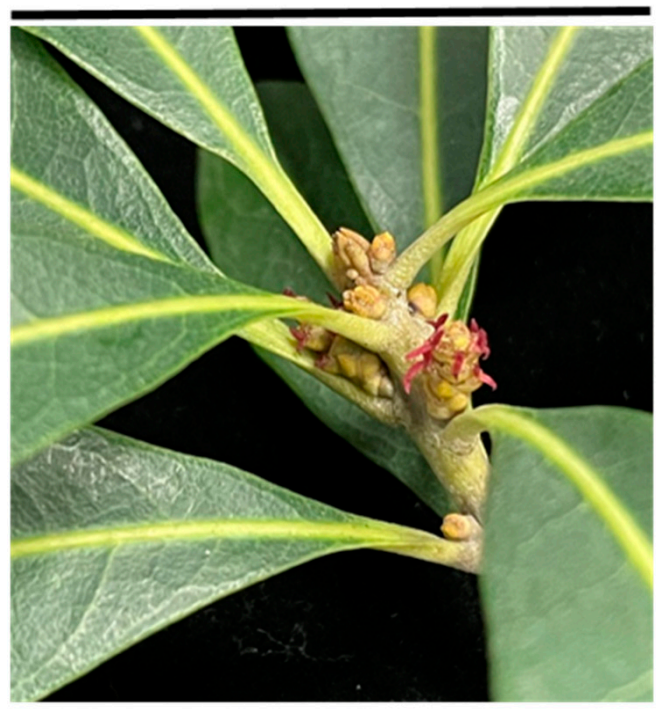

C

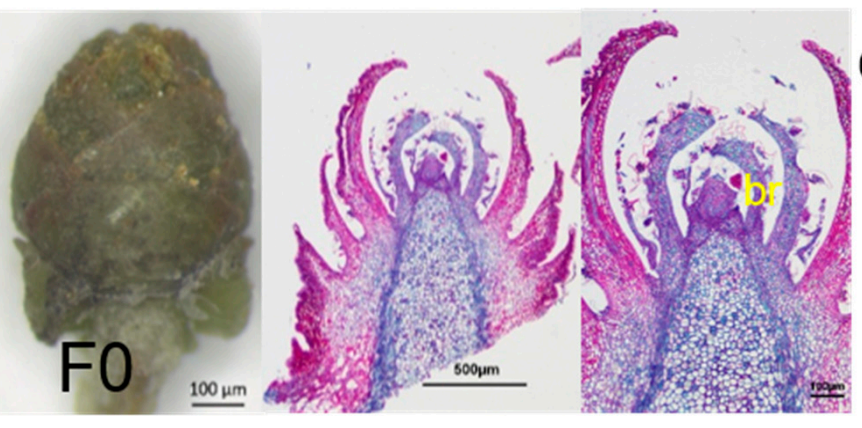

e

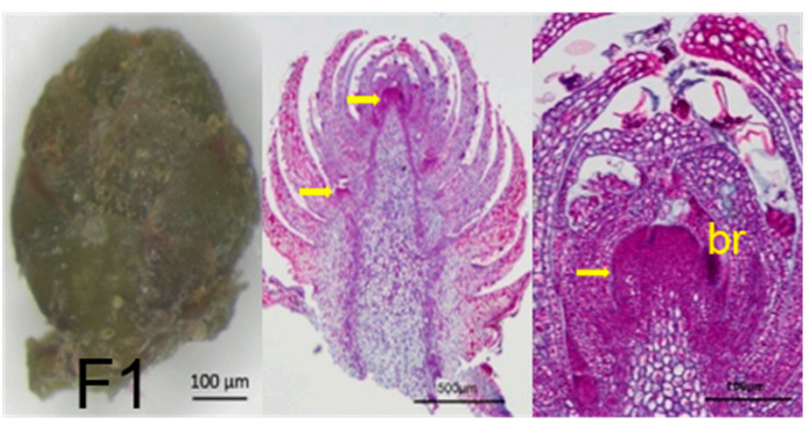

- Female primordium
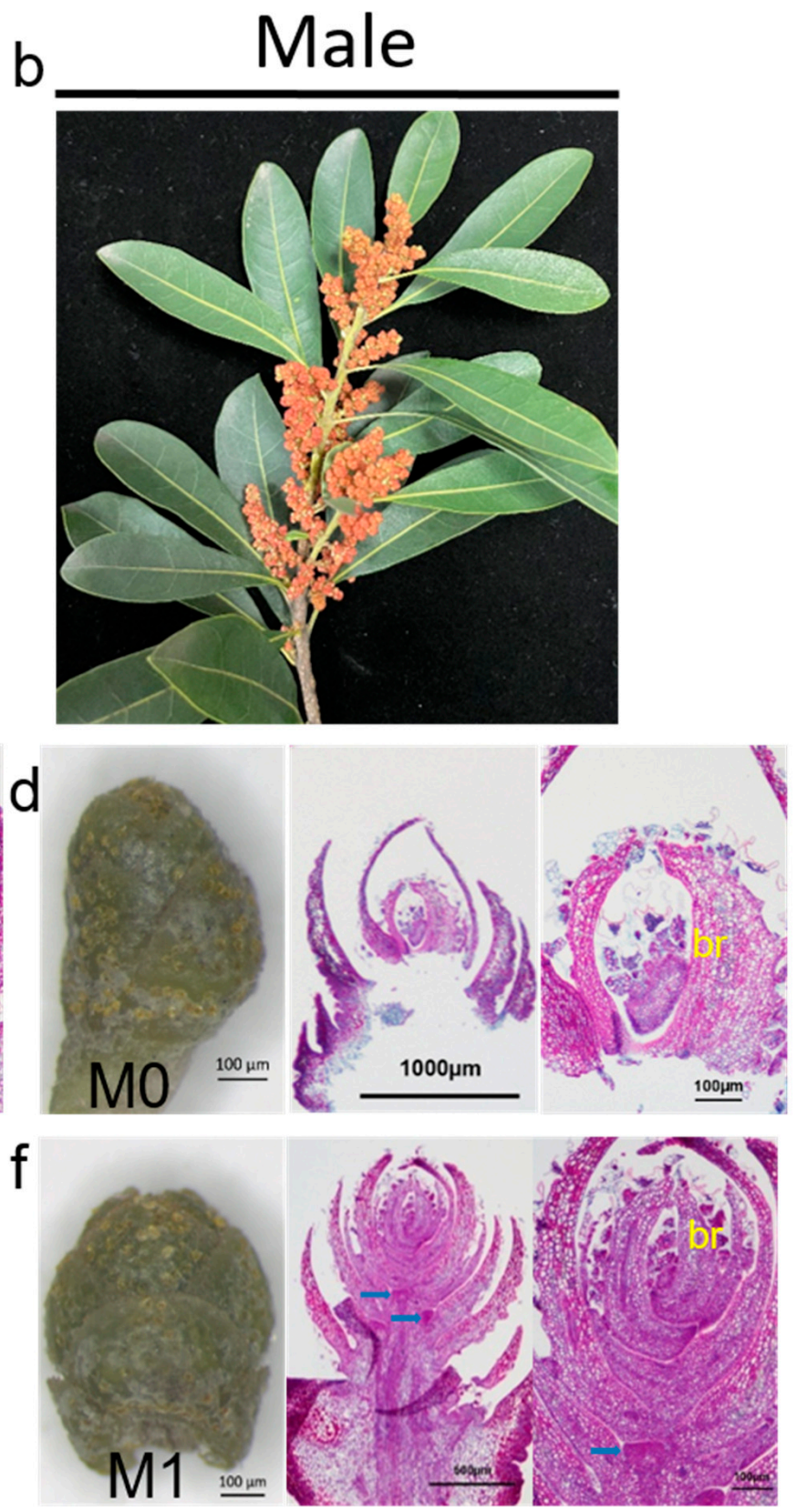

$\rightarrow$ Male primordium

Figure 1. The male and female floral buds of the red bayberry. Female flower (a) and male flower (b) of the red bayberry. Male and female floral buds before sexual differentiation (c,d), and with female and male primordia $(\mathbf{e}, \mathbf{f})$. br, bracts. The figures $(\mathbf{e}, \mathbf{f})$ have been published in a previous study [7].

\section{Materials and Methods}

\subsection{Flower Sample Collection and Morphological Observation}

The male accession 'H2011-12' of a red bayberry, an approximately 30-year-old tree which was used for whole-genome sequencing [7], and the female individual 'H2011-11', grown at Zhejiang University Campus (Hangzhou, China), were used for all experiments. 
Floral buds were collected on two dates in 2017: 13 July and 27 August. The male and female floral buds were sampled in three replicates. The samples were immediately frozen in liquid nitrogen and stored at $-80{ }^{\circ} \mathrm{C}$ prior to RNA extraction. The male and female flowers were screened under a stereoscopic microscope. Paraffin sections were used as in a previous study [29] to confirm the developmental stages of male and female buds. We used floral buds before sexual differentiation (F0 for female and M0 for male floral buds) and the early emergence of female and male primordia (F1 for female and M1 for male floral buds) for transcriptomic analysis to uncover the molecular regulatory mechanisms of unisexual flower development and sexual differentiation in the red bayberry.

\subsection{RNA Extraction and Transcriptome Sequencing}

The total RNA of male and female flowers was extracted by using a Quick RNA isolation Kit (Waryong, Beijing, China) according to the manufacturer's manual. The quality of total RNA was checked on 1\% denatured agarose gel and then assessed on an Agilent Bioanalyzer 2100 system (Agilent Technologies, Santa Clara, CA, USA). The pair-end cDNA sequencing libraries of four floral buds were prepared with three biological replicates by the Beijing Genome Institution (BGI, Beijing, China) and then sequenced at BGI using an Illumina HiSeq 2500 platform (Illumina) with PE125.

\subsection{RNA-Seq Data Analysis}

Adapter sequences, ambiguous nucleotides, and low-quality sequences in raw reads were removed with SOAPnuke (version1.4.0) [30] and Trimmomatic (v0.36) [31]. The paired-end clean reads from each library were mapped to the reference genome (https: / / www.ncbi.nlm.nih.gov / genome/?term=Morella+rubra, accessed on 15 February 2022) using HISAT2 (Hierarchical Indexing for Spliced Alignment of Transcripts) to calculate the mapping ration [32]. Clean reads were aligned to complete the reference using Bowtie2 [33], and RSEM (RNA-Seq by Expectation Maximization) was used to estimate the abundance of the genes [34]. Read counts were normalized by calculating the FPKM (Fragments Per Kilobase of exon model per Million mapped fragments) value of all the transcripts in the samples. Gene function was annotated based on NCBI nonredundant protein sequences $(\mathrm{Nr})$, the protein family (Pfam), the Swiss-Prot protein database and the Kyoto Encyclopedia of Genes and Genomes (KEGG). Gene ontology (GO) annotation was used for sequences with a match in the Nr database by using Blast2GO v.3.0 [35]. Differential expression between the two groups was analyzed with the DESeq R package version 1.30.0 [36]. Genes with a minimal two-fold difference in expression ( $\mid \log 2$ Ratio $\mid \geq 1$ ) and padj $\leq 0.01$ were considered to be differentially expressed genes (DEGs). Heatmaps (scaled by row) were prepared with TBtools and used for GO enrichment analysis and KEGG pathway enrichment analysis [37]. For the weighted gene co-expression network analysis (WGCNA), an R package (version 1.0.7) [38] was used to construct a potential regulatory network of sex differentiation in the red bayberry.

\subsection{Validation of RNA-Seq Data}

Quantitative real-time PCR (qRT-PCR) was used to validate the RNA-seq data. The Total RNA of F0, F1, M0 and M1 were extracted by using the Quick RNA isolation Kit (Waryong, Beijing, China) according to the manufacturer's manual, and the concentration was checked on a BioDrop spectrophotometer (Biochrom, Cambridge, UK). First-strand cDNA synthesis was performed with $2 \mu \mathrm{g}$ RNA with EasyScript ${ }^{\circledR}$ All-in-One First-Strand cDNA Synthesis SuperMix for qPCR (One-Step gDNA Removal) (TransGen Biotech, Beijing, China). The qRT-PCR primers were designed using an online tool, Primer 3 (https:// primer3.ut.ee/, accessed on 15 February 2022). The PCR products of qRT-PCR were verified by sequencing. The qPCR was performed with the SYBR Premix Ex Taq ${ }^{\mathrm{TM}}$ (Takara, Ohtsu, Japan) and CFX Connect ${ }^{\mathrm{TM}}$ real-time PCR system (Bio-Rad, Hercules, CA, USA) with the reaction and program detailed as previously published by Ni et al. [39]. Actin 
was used for normalization, and the expression data were calculated with the $2^{-\Delta \Delta \mathrm{Ct}}$ formula [40].

\section{Results}

\subsection{Morphological Observation and Dissecting Red Bayberry Flower Buds}

Red bayberry floral meristems were initiated in the bract axils of both male and female plants. At the early stage of floral development, the inflorescence rachis became elongated (Figure 1c,d), and this was followed by the flower initiation stage, with the female and male flower primordia forming between the two bracts (Figure 1e,f). At this stage, there was no obvious morphological difference between male and female flowers.

\subsection{Sequencing of Red Bayberry Flower Buds}

A total of 562.3 million raw sequencing reads were obtained (Table S1), and the clean reads were aligned to the red bayberry reference genome and used to quantify the expression levels of genes using HISAT and Bowtie2. About $83.5 \%$ of reads could be mapped to the reference genome (Table S1). A principal component analysis (PCA) based on FPKM values separated the samples into four distinct groups, with each sample making a separate group with its replicates (Figure S1). The total variation portion $(46.2 \%)$ consists of two principal components (PC1: 27.6\%, PC2: 18.6\%), with PC1 separating females from males. The distributions of the number of genes at different expression levels among the four groups were similar (Figure 2a). The number of genes not expressed (FPKM $<1$ ) accounted for the highest proportion, followed by those genes expressed at a low level $(1 \leq$ FPKM $<10)$ and mid-level $(10 \leq$ FPKM $<100)$. The highly expressed genes with FPKM $\geq 100$ accounted for the smallest proportion. About $85 \%$ of genes were expressed in both female and male floral buds, and 1979 genes were specifically expressed in female and 2015 in male buds (Figure 2b).

\subsection{Gene Ontology (GO) and KEGG Enrichment Analysis of Highly Expressed Genes Revealed Rapid Growth in Female and Male Buds}

A total of 633, 677, 521 and 558 highly expressed genes were detected in F0, F1, M0 and M1 floral buds, respectively, and $61.3 \%$ of them were shared between female and male floral buds (Figure 2c). The GO enrichment analysis and KEGG pathway enrichment analysis of the highly expressed genes in female and male floral buds were performed. The results from the GO enrichment analysis of the highly expressed genes showed that genes involved in cell proliferation, such as the 'structural constituent of ribosome' and 'structural molecule activity' belonging to molecular function categories, 'ribosomal subunit' and 'ribosome' belonging to the cellular component and the 'peptide biosynthetic process' and 'peptide metabolic process' belonging to the biological process, were more abundant (Table S2). This was in agreement with the results of the KEGG enrichment analysis, with ribosome overrepresented in the four floral samples (Table S3). In these analyses for midlevel expressed genes in four floral samples (Tables S4 and S5), the KEGG enrichment results indicated that ribosome was overrepresented in the four floral samples (Table S5). These results suggest that floral buds at these two development stages undergo rapid cell division and expansion.

\subsection{Identification of Differentially Expressed Genes}

We compared female vs. male floral buds in pair-wise analyses of M0 vs. F0 and M1 vs. F1. We also compared floral buds within a sexual type, F1 vs. F0 and M1 vs. M0. In all, 8889 genes were significantly expressed, with $|\log 2 \mathrm{FC}| \geq 1$ and $p$-value $<0.01$ in four pairs (Figure S2), including 4970 DEGs in M0 vs. F0, 5421 DEGs in M1 vs. F1, 2659 DEGs in F1 vs. F0 and 2888 DEGs in M1 vs. M0 (Figure 2d). The number of DEGs between female and male was greater than those within one sex in an inner comparison, suggesting that a very complex physiological and biochemical process occurs during sexual differentiation and unisexual flower development. 
a

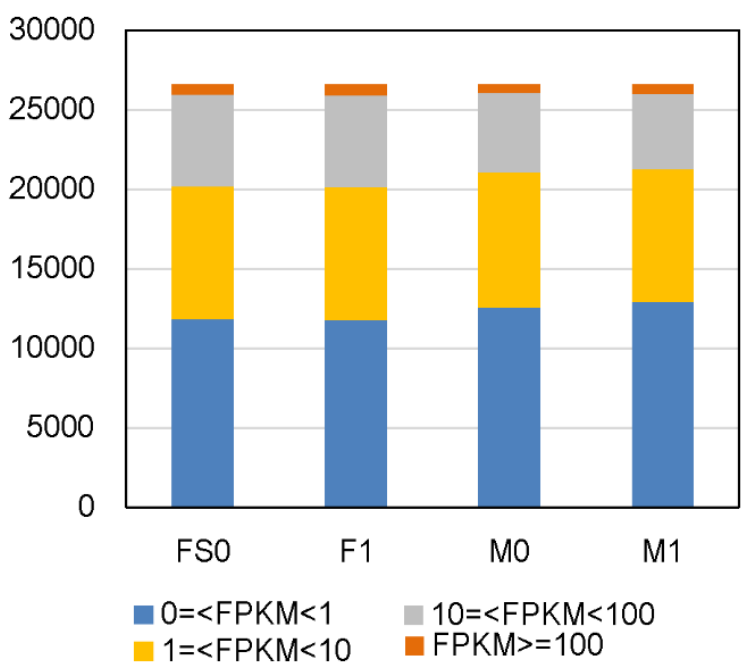

C

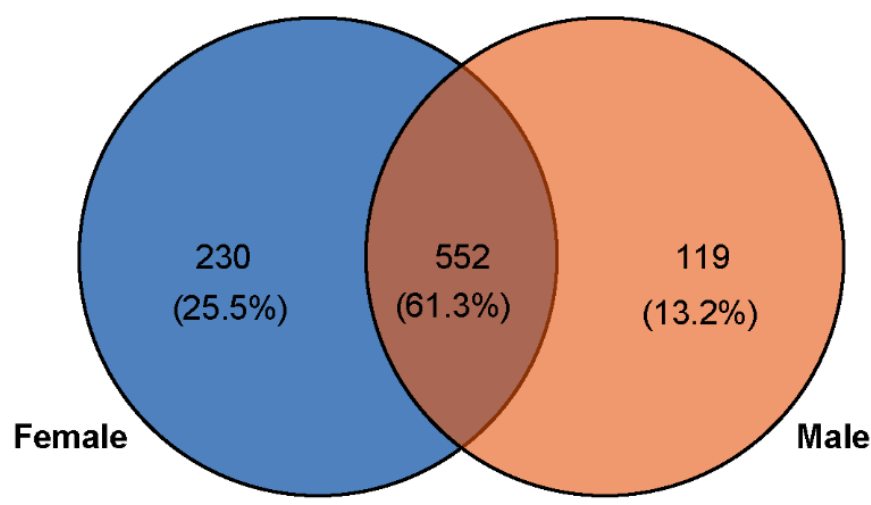

b



d

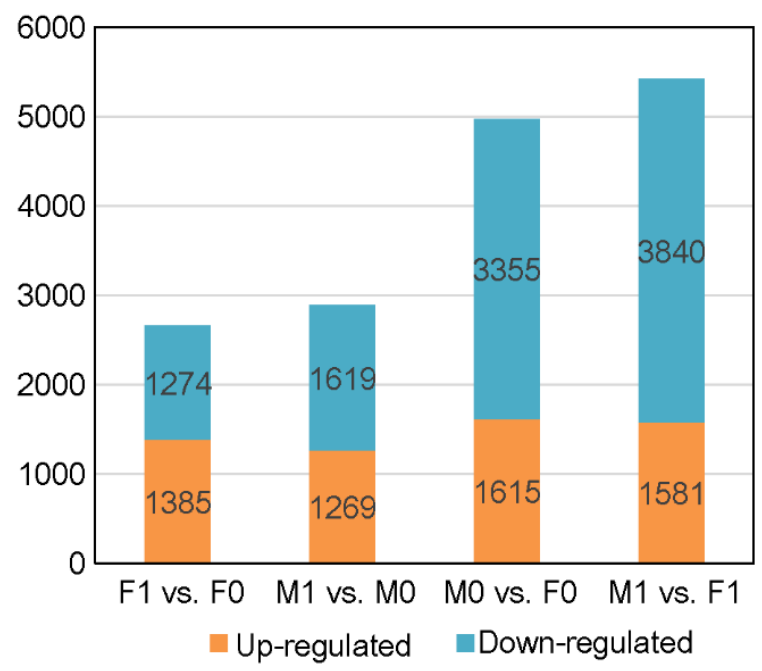

Figure 2. Overview of red bayberry M0, M1, F0 and F1 floral bud transcriptome. (a) Distribution of genes with high expression (FPKM $\geq 100)$, mid-level expression (100 > FPKM $\geq 10)$, low expression $(10>$ FPKM $\geq 1)$ and no expression $(1>$ FPKM $\geq 0)$. Venn diagram of genes expressed in female and male libraries (b) and genes with high expression in at least one library (c). (d) Distribution of upand down-regulated DEGs in the pair-wise F1 vs. F0, M1 vs. M0, M0 vs. F0 and M1 vs. F1 analyses.

Because we aim to identify genes related to sexual differentiation in the red bayberry, we only focus on an analysis of DEGs between M0 vs. F0 and M1 vs. F1. We further used GO and KEGG enrichment to analyze DEGs between M0 vs. F0, M1 vs. F1, and all the 7029 DEGs of the two compared groups. GO enrichment results showed that catalytic activity was overrepresented in molecular functions (Table S6). KEGG pathway enrichment analysis showed that plant hormone signal transduction (ko04075) was significantly overrepresented in M0 vs. F0 and M1 vs. F1 and all 7029 DEGs (Figure S3), suggesting that plant hormones play a role in sex differentiation in the red bayberry. A total of 91 genes were identified in this category, 35 associated with auxin, 14 involved in CK, 10 with ABA and JA, and the remainder associated with gibberellin, BR, ethylene, and SA (Table S7).

\subsection{DEGs Are Involved in Plant Hormone Biosynthesis and Signalling}

To investigate hormone function during the process of sexual differentiation, hormonerelated genes were identified in the red bayberry. A total of 161 hormone-related genes were 
identified in 7029 DEGs (Table S8), and the distribution for different hormones is shown in Figure 3a: 42 genes related to auxin were most represented, followed by 26 related to ethylene, 20 to $\mathrm{CK}, 18$ to SA, 17 to ABA, 15 to GA and JA, and 8 to brassinosteroid. Among the 161 hormone-related genes, 62 DEGs were significantly up-regulated in females and 29 were in males (Table S8). Moreover, a total of 18 genes displayed female-specific expression and 10 genes were expressed only in males (Table S9).

a

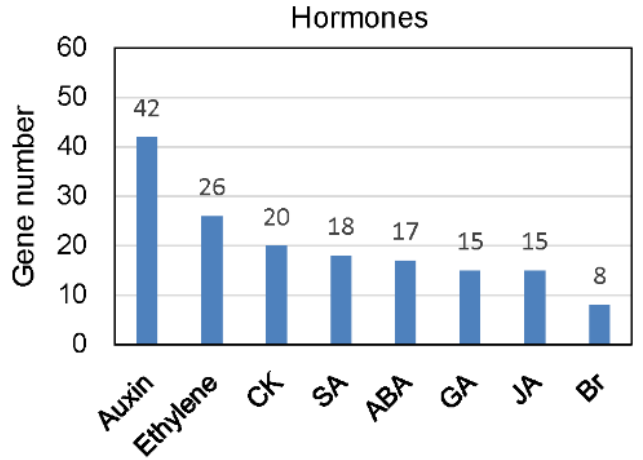

b


Figure 3. The distribution and expression of hormone-related genes. (a) The distribution of 184 hormone-related genes. (b) Heat map of sex-biased DEGs related to hormones, with the FPKM value of genes shown in the box.

The expression of 13 out of 42 auxin-related genes was significantly up-regulated in females. MR1G009013.1 (SAUR) was expressed at a much higher level in females than in males (Figure $3 b$ ). Five of them were only expressed in females, including three $A R F$ genes: MR3G011095.1, MR4G010547.2 and MR7G007520.1; one IAA gene MR3G001167.1 and one YUC gene MR1G027448.1 (Table S9). The expression of six auxin-related genes was significantly up-regulated in males, including the GH3.9 gene MR8G002059.1, which was only expressed in males. In the present study, 12 out of 26 ethylene-related genes were significantly up-regulated in females, including 4 female-specific expressed genes, MR1G003948.1 (ETO1), MR2G007010.1 (ERF4), MR2G016358.1 (ETO1), and MR3G008357.1 
(ACO) (Figure 3b and Table S9). The ethylene biosynthesis gene ACO (MR3G008356.1) and ethylene signal transduction gene ERF5 (MR4G016016.1) were expressed at a much higher level in females than in males (Figure 3b). Four ethylene-related genes were significantly up-regulated in the male buds, including three male-specific expressed genes: MR0TCONS_00017483.1 (ACO), MR6G009324.1 (ACO) and MR2G016675.1 (EIN2). The jasmonic acid biosynthesis gene MR6G021641.1 (AOC) also had male-specific expression (Figure 3b). The cytokinin biosynthesis gene MR0G027265.2 (LOG) and signal transduction gene MR3G019062.1 (CYCD3-1) were only expressed in females, while two UGT genes involved in SA biosynthesis gene had the opposite expression pattern: MR2G009173.1 was only expressed in females and MR4G020673.1 was only expressed in males. The brassinosteroid signal transduction gene BIM1 (MR5G011875.1) showed male-specific expression and BAK (MR7G008146.2) had much higher expression in females than in males (Figure 3b).

\subsection{Transcription Factors Differentially Expressed in Male and Female Buds}

Transcription factors (TFs) play important roles in flower development. A function annotation of the 7029 DEGs revealed that 415 transcription factors belong to 55 gene families (Table S10). The MYB family, AP2/EREBP family and bHLH family were over-represented (Figures 4a and S4). Among the transcription factors, the expression of 129 transcription factors genes were significantly up-regulated in females, and 53 were down-regulated. On further analysis of the expression of the sex-type-specific genes, 38 were found to be only expressed in females and 18 in males (Table S11).

Of the 58 MYB family genes, there was a significant female-biased expression of 12, and 6 had significant male-biased expression. The MR4G023331.1 and MR4G009025.1 gene expression was female-specific, while the MR1TCONS_00020658.1 was only expressed in males; its homolog in Arabidopsis AT3G13540.1 (AtMYB5) is involved in flavonoid biosynthesis. Among 36 genes in the bHLH gene family, 6 genes expressed were female-specific, including MR1G020885.1, MR6G026964.1, MR7G020473.2, MR2G024394.1, MR4G009030.1 and MR8TCONS_00067952.1, and 3 genes were male-specific, including MR5G011875.1, MR6G001563.1 and MR8G020751.1 (Figure 4b). Four of AP2/EREBP genes displayed female-specific expression, including MR0G006452.1, MR1G017646.1, MR2G007010.1 and MR8G022041.1. Moreover, MR2G007010.1 was highly expressed in females with FPKM values of 357 and 260 for F0 and F1, respectively (Figure 4b).

MADS-box genes are involved in floral development and floral organ identity. In this study, we identified 10 MADS-box genes differentially expressed in the two groups. The SOC1 gene MR0G005926.1 was only expressed in males (Figure 4b); SOC1 activates the floral meristem identity gene LEAFY (LFY). Two C2C2-CO-like zinc finger genes, MR6G018079.1 and MR3G018958.1, were specifically expressed in females, and MR8G027384.1 was expressed only in males. We also found that two C2C2-Dof genes, MR5G011870.1 and MR3G009872.2, and one C2C2-GATA gene, MR6G010822.1, were only expressed in females.

Among the 415 transcription factors, the expression of 24 of them was high or medium in females and were not expressed in males (Figure 4b). The hormone-related MYC, MYB, STRE, ABRE and TF transcription factor binding sites in the promoters were also enriched (Table S12).

\subsection{Genes in the Non-Recombining Region of the Red Bayberry W Chromosome}

The $59 \mathrm{~kb}$ FSR (female-specific region) had seven predicted genes, and the expression levels of seven female-specific genes are shown in Table 1 . The expression of all the femalespecific genes in F0 and F1 was very low, with the FPKM value < 2, except for MrTFIID2. The expression of these genes was consistent with those previously reported in red bayberry floral buds using qRT-PCR [7], and further confirmed that the gene expression profile of the RNA-seq data was reliable. 
a

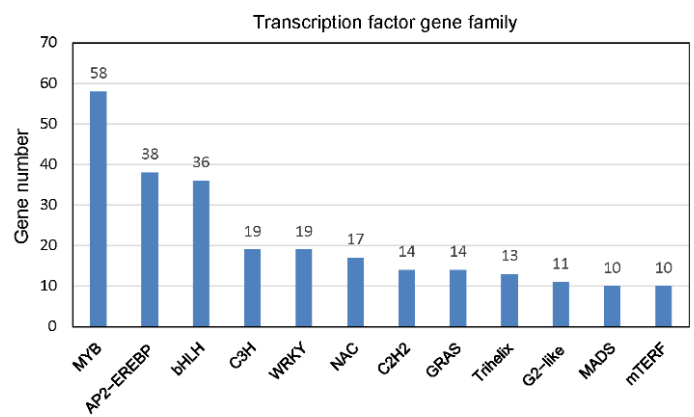

b



Figure 4. The distribution and expression of transcription factor genes in all 8889 DEGs. (a) The distribution of 547 transcription factor genes (top 12 families). (b) Heat map of sex-biased DEGs related to transcription. The FPKM value of genes is shown in the box.

Table 1. List of the expression level of seven female-specific genes in red bayberry floral buds.

\begin{tabular}{cccccc}
\hline Gene Name & Gene_Id & M0_FPKM & M1_FPKM & F0_FPKM & F1_FPKM \\
\hline MrCPS2 & MR8G025874.1 & 0 & 0 & 0.00 & 0.04 \\
MrCKA2 & MR8G025875.1 & 0 & 0 & 0.71 & 0.60 \\
MrTFIID2 & MR8G025876.1 & 0 & 0 & 4.90 & 4.33 \\
MrASP2 & MR8G025877.1 & 0 & 0 & 0.53 & 1.14 \\
MrSAUR2 & $M R 8 G 025878.1$ & 0 & 0 & 0.44 & 2.06 \\
MrLsd90-2 & $M R 8 G 025879.1$ & 0 & 0 & 0.43 & 0.59 \\
MrFT2 & $M R 8 G 025880.1$ & 0 & 0 & 0.23 & 0.25 \\
\hline
\end{tabular}

\subsection{Co-Expression Networks of Female and Male Buds}

We used weighted gene co-expression network analysis (WGCNA) [38] to construct a potential regulatory network of sex differentiation in the red bayberry. A total of 42 modules were established using WGCNA in four male and female flower buds at early development stages (Figure 5a). The genes in the same module had strong connectivity (Figure 5b). The expression pattern of each module was analyzed in all samples. The module-trait relationships showed that the light-green $\left(r=0.97, P=6 \times 10^{-5}\right)$ and dark-cyan $(r=-0.75, P=0.03)$ modules were highly associated with the sex phenotype (Figure $5 c$ ), so these two modules may play important roles in regulating sex differentiation and flower development. 


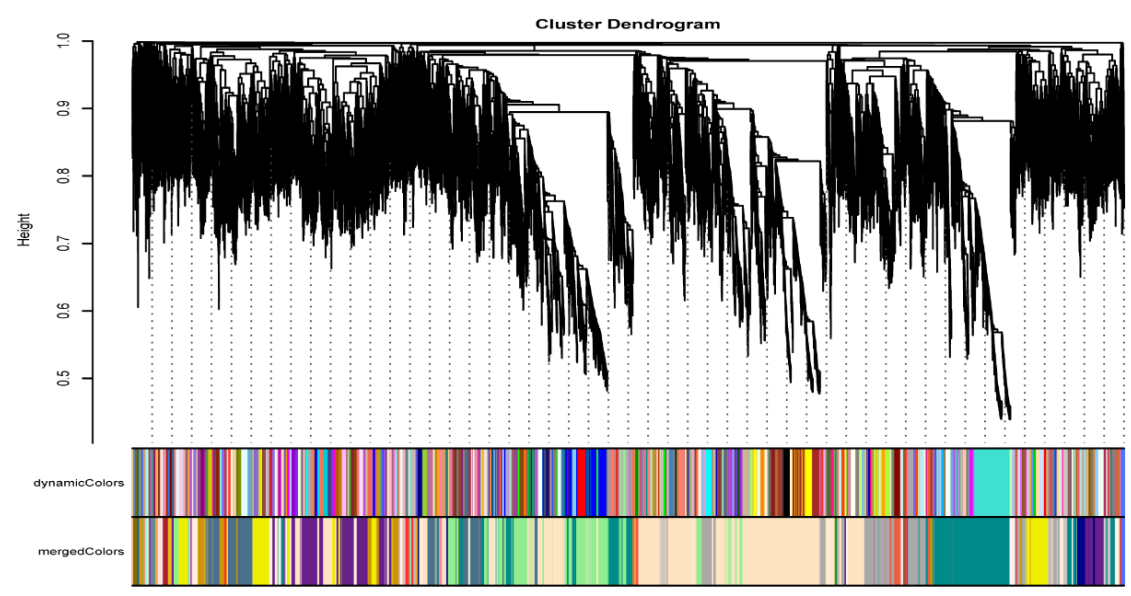

b


Figure 5. Weighted gene co-expression network analysis. (a) Hierarchical cluster tree showing co-expression modules identified using WGCNA; 'DynamicColors" represents modules divided according to clustering results, and "MergedColors" represents the merging of the module with a similar expression pattern according to the module similarity, with the analysis conducted according to the merged module. (b) Module gene correlation analysis; each row and column represent a gene; the darker the color of each point indicates a higher connectivity between the two genes. (c) Sample expression pattern analysis. 
We screened the genes related to hormone and transcription factors in the two modules (Table S13). A total of 55 DEGs were selected from the light-green and 126 DEGs from the dark-cyan modules. As shown in Figure 6a and Table S14, the hub gene in the light-green module was MROTCONS_00017483.1, which encodes 1-aminocyclopropane1-carboxylate oxidase (ACO), which is a key gene in ethylene biosynthesis, and is only expressed in males (Figure 3b). In addition, the module included genes involved in ethylene biosynthesis (MR2G016358.1 (ETO1), which is only expressed in females (Figure 3b)), ABA signaling (MR8G022503.2 and MR7G016748.1 (PP2C)) and CTK signaling (MR7G024824.1 $(A R R 5)$, a male-specific expressed gene (Figure 6a)). Nine transcription factors were identified in the light-green module co-expression network, including three zinc finger genes (MR5G011870.1 (Dof), MR8G027384.1 (COL4) and MR1G019332.1 (C3H)), one MYB gene (MR1TCONS_00020658.1), one AP2/EREBP (MR2G007010.1), one PHD gene (MR5G024537.1), one Tify (MR8G021808.1), one GRF (MR4G021188.1) and two bHLH genes (MR6G001563.1 and MR8G020751.1). The floral meristem determinacy gene MR6G008503.1 (ULT1) was also in the light-green module co-expression network (Figure 6a and Table 2).

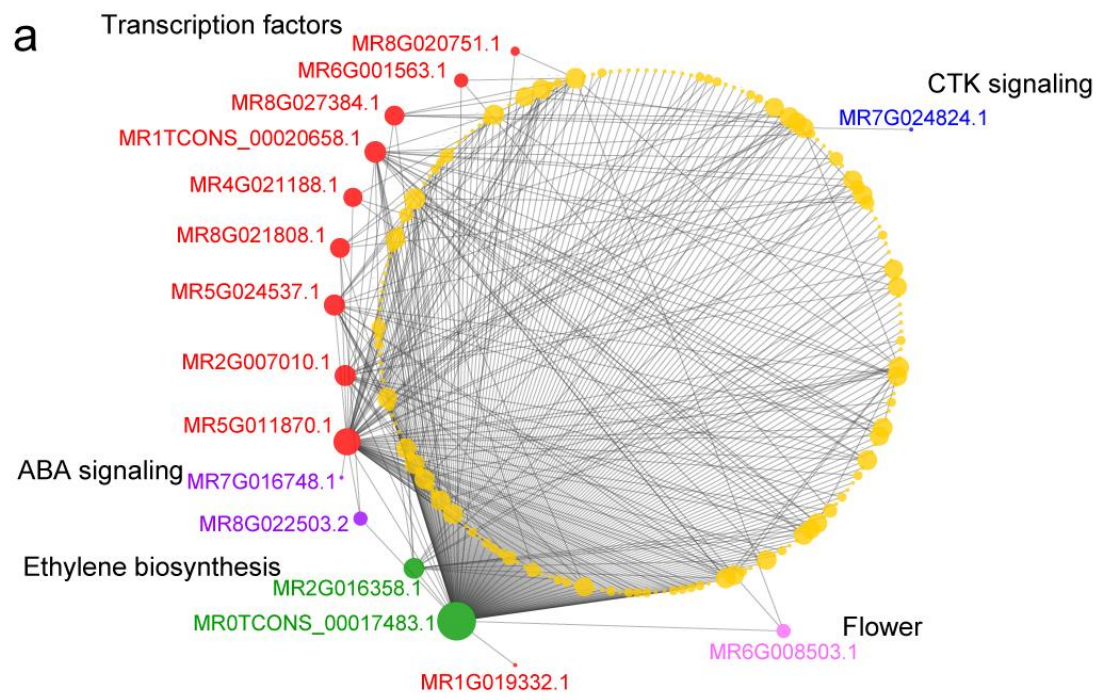

b

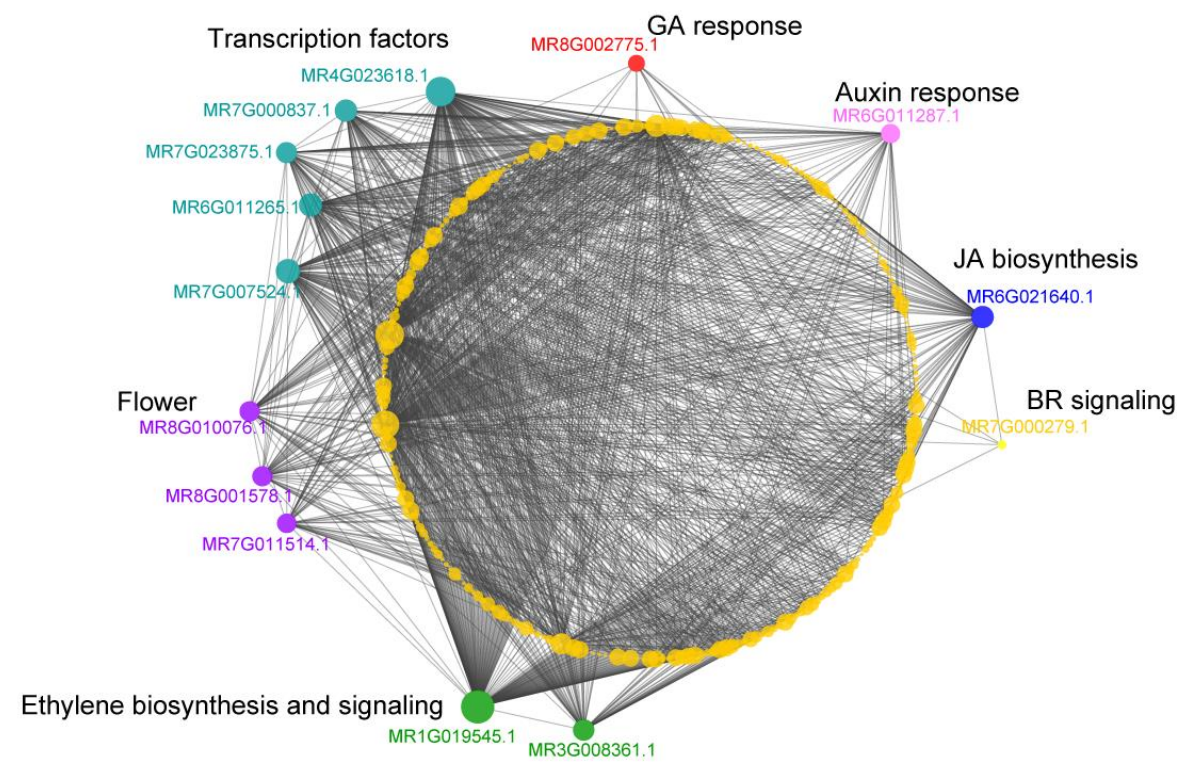

Figure 6. Visualization of co-expression network. (a) Co-expression network of genes in light-green modules. (b) Co-expression network of genes in dark-cyan modules. 
Table 2. List of hormone-related genes, transcription factors and flowering-related genes in two modules.

\begin{tabular}{|c|c|c|c|c|}
\hline Module & Gene ID & Gene Name & Specifically Expressed in & Involved Process \\
\hline \multirow{16}{*}{ Light green } & MR0TCONS_00017483.1 & $\mathrm{ACO}$ & male & \multirow{2}{*}{ ET biosynthesis } \\
\hline & MR2G01̄6358.1 & ETO & female & \\
\hline & MR8G022503.2 & PP2C & & \multirow{2}{*}{ ABA signaling } \\
\hline & MR7G016748.1 & PP2C & & \\
\hline & MR7G024824.1 & ARR5 & male & CTK signalling \\
\hline & MR5G011870.1 & dof & female & \multirow{10}{*}{ Transcription factor } \\
\hline & MR1TCONS_00020658.1 & MYB & male & \\
\hline & $M R 2 G 007010.1$ & AP2/EREBP & female & \\
\hline & MR5G024537.1 & TAZ & female & \\
\hline & MR8G021808.1 & Tify & female & \\
\hline & MR8G027384.1 & C2C2-CO-like & male & \\
\hline & MR4G021188.1 & GRF & & \\
\hline & MR6G001563.1 & bHLH106 & male & \\
\hline & MR8G020751.1 & bHLH106 & male & \\
\hline & MR1G019332.1 & $\mathrm{C} 3 \mathrm{H}$ & & \\
\hline & MR6G008503.1 & ULT & male & Flower development \\
\hline \multirow{14}{*}{ Dark cyan } & MR1G019545.1 & ETR & - & FT hiocunthesis and sionaling \\
\hline & MR3G008361.1 & $\mathrm{ACO}$ & & E I biosynthesis and signaling \\
\hline & MR8G001578.1 & SPL & & \multirow{3}{*}{ Flower development } \\
\hline & MR7G011514.1 & VRN1 & & \\
\hline & MR8G010076.1 & UGT & & \\
\hline & MR6G021640.1 & $\mathrm{AOC}$ & & Jasmonic acid biosynthesis \\
\hline & MR6G011287.1 & AUX22D & & Auxin response \\
\hline & MR8G002775.1 & GASA1 & & GA response \\
\hline & MR7G000279.1 & ASK7 & & Gibberellin \\
\hline & MR7G007524.1 & AP2-EREBP & & \multirow{5}{*}{ Transcription factor } \\
\hline & MR4G023618.1 & $\mathrm{C} 3 \mathrm{H}$ & & \\
\hline & MR7G000837.1 & ZF-HD & & \\
\hline & MR7G023875.1 & ZF-HD & & \\
\hline & MR6G011265.1 & bzip & & \\
\hline
\end{tabular}

The hub genes in the dark-cyan co-expression network include MR1G019545.1, which encodes ethylene response 1, with its homolog AtETR1 (AT1G66340), which is involved in ethylene signaling pathways, and MR4G023618.1, which encodes the $\mathrm{C} 3 \mathrm{H}$ zinc finger transcription factor. The dark-cyan module also included five hormone-related genes, four transcription factors and three flower development genes (Figure 6a and Table S15). The details are shown in Table 2: MR3G008361.1 (ACO) is involved in ethylene biosynthesis; MR6G021640.1 (AOC) is involved in JA biosynthesis; MR7G000279.1 (ASK7) is involved in the brassinosteroid signal transduction pathway; and MR6G011287.1 (AUX22D) and MR8G002775.1 (GASA1) are involved in the auxin and gibberellin response, respectively. With regard to the transcription factors, MR7G007524.1 belongs to the AP2/EREBF gene family and MR6G011265.1 encodes the bZIP transcription factor. MR7G000837.1 and MR7G023875.1 belongs to the zinc finger gene family. There were three genes related to flower development: MR8G010076.1, which encodes homologs of AtUGT87A2 (AT2G30140), which regulate flowering time via FLOWERING LOCUS C; MR7G011514.1 the homolog of Arabidopsis AtVRN1; and the MR8G001578.1 homolog in Arabidopsis is AtSPL14 (AT1G20980). These results showed that ethylene biosynthesis and signaling pathways may play vital roles in sex differentiation and flower development, with the transcription factor playing vital roles in regulating flower buds. 


\subsection{Validation of Gene Expression Level in Male and Female Red Bayberry Floral Buds}

To confirm the gene expression pattern of the male and female floral buds identified in the RNA-Seq data, ten DEGs were randomly selected for validation by using qRT-PCR and the primers were shown in Table S16. The results of the qPCR were consistent with the gene expression patterns from RNA-seq (Figure S5), which confirmed that the gene expression profile of the RNA-seq data was reliable.

\section{Discussion}

In the present study, we constructed female and male Morella rubra floral bud libraries representing two early development stages, and used transcriptomes to analyze the genes differentially expressed in female and male floral buds to identify sex differentiation- and flower development-related genes.

The identification of sex-biased genes related to flower development is helpful to reveal the sex differentiation mechanisms. Here, we found 8889 DEGs in pair-wise analyses of F1 vs. F0, M1 vs. M0, M0 vs. F0 and M1 vs. F1 that may be related to sex differentiation and flower development. KEGG analysis indicated these DEGs were functionally enriched in plant hormone signal transduction (ko04075) (Figure S3). Phytohormones regulate various developmental processes, including floral development and sex differentiation, and the effects of plant hormones on sex differentiation vary among plant species [41]. Ethylene has been demonstrated to play a key role in the sex determination of cucumber and melon $[15,42,43]$. In maize, GA suppresses stamen development, while brassinosteroid and jasmonate coordinately suppress tassel development $[44,45]$. The exogenous application of ethrel, an auxin inhibitor, can induce male-to-female sex reversal in papaya [24]. In the red bayberry, the exogenous application of a GA inhibitor can induce female-to-male sex reversal in the 'Dongkui' and other cultivars (http:/ / patents.google.com/patent/CN10758 1062A/en, accessed on 15 February 2022).

Hormones can crosstalk to regulate floral organ development. For example, ethylene can control floral transition via the DELLA-dependent regulation of floral meristem identity genes LEAFY (LFY) and SUPPRESSOR OF OVEREXPRESSION OF CONSTANS 1 (SOC1) [46], and salicylic acid regulates flowering [47,48]. In this study we found 161 DEGs that were related to hormones, with 18 female-specific expressed genes and 10 male-specific expressed genes. Those genes, for instance, ethylene-related genes MR2G016358.1 (ETO1) and MROTCONS_00017483.1 (ACO), are good candidates for studying the mechanism of red bayberry sex differentiation.

That transcription factors play vital roles in the regulation of sex differentiation and flower development has been reported in many plants. In the present study, we identified 415 transcription factors among the 7029 DEGs. Transcription factors interact with hormone-related genes to control sex identity. Moreover, the rest of three male-specific expressed genes, including one MYB gene (MR1TCONS_00020658.1) and two BHLH genes (MR6G001563.1 and MR8G020751.1), have been reported to regulate flavonoid biosynthesis. Male floral differentiation has been found to be significantly related to flavonoid biosynthesis in Jatropha curcas L., and the inhibition of flavonoid biosynthesis causes male sterility in Petunia $[27,49]$. Our results suggest that transcription factors related to flavonoids may play roles in male floral differentiation.

For example, the sex G (gynoecious) gene CmWIP1, a C2H2-type zinc finger transcription factor, is a central integrator of the transcriptional networks, leading both to the inhibition of carpel development and the control of the expression of the stamen inhibitor CmACS-7 in melon [50]. The female-specific expressed gene MR2G002020.1 encodes the WIP2 protein identified in the present study. Based on the WGCNA results, a total of 15 transcription factors were identified, including four genes specifically expressed in females and four in males (Table 2). The homologue of the male-specific gene MR8G027384.1 in Arabidopsis is COL4 (AT5G24930.1), which can regulate flowering time [51]. The homolog of the zinc finger gene MR3G018958.1, also specifically expressed in the female (encoding a CONSTANS-like protein), in rice is OsCOL16, which represses flowering by up-regulating 
Ghd7 expression [52]. We also identified the male-specific expression of certain genes. The homologs of the MADS-box gene MR0G005926.1 in Arabidopsis is SOC1, which is a core gene in the floral induction pathway $[53,54]$. Those sex-specific expressed transcription factor genes may play a role in the induction of female and male primordia in the red bayberry. Clearly, this sex-biased expression of transcription factors may interact with other genes to regulate red bayberry sex differentiation, and we will verify their function in future research.

To narrow down the key genes regulating sex differentiation in the red bayberry, we constructed the regulation network of sex differentiation in the red bayberry using WGCNA (Figures 5 and 6). Interestingly, we found modules coded light green and dark cyan to be associated with sex type. Both hub genes in the two modules were ethylene-related genes, and the homolog of MROTCONS_00017483.1 in the light-green module network was AtACO, which is a key gene in ethylene biosynthesis. Moreover, MROTCONS_00017483.1 was expressed specifically in the male bud. In the dark-cyan module network, MR1G019545.1 is homologous to AtETR1, which is involved in ethylene signaling pathways (Figure 6 and Table 2).

Sex differentiation genes have been found to be involved in ethylene biosynthesis and signaling pathways in Cucurbitaceae species. In the cucumber, sex differentiation is mainly controlled by three ACS (1-aminocyclopropane-1-carboxylate (ACC) synthase) genes, including the $F(C s A C S 1 G), M(C s A C S 2)$ and $A$ (CsACS11) genes. Additionally, $A C O$ is also essential for the development of female flowers [18]. A recent study has shown that the ethylene receptors CpETR1A and $C p E T R 2 B$, highly homologous to AtETR1 and AtETR2, cooperate in the control of sex determination in Cucurbita pepo [55]. In present study, the male-specific expressed gene MROTCONS_00017483.1 (ACO) and female-specific expressed gene MR2G016358.1 were identified in light-green module. Therefore, we can speculate that ethylene may be the key hormone regulating red bayberry male and female flower differentiation. We found GA-, auxin-, ABA-, CTK-, SA- and Br-related genes in both modules and transcription factors. We also identified three genes involved in floral identity and development. The homolog of MR8G010076.1 is AtUGT87A2, which can regulate flower time via FLC (FLOWERING LOCUS C) [56], while the MR7G011514.1 homolog in Arabidopsis, AtVRN1, acts as a key gene in the vernalization pathway, mediated by the floral pathway integrator FT (FLOWERING LOCUS T) and targeted to FLC to regulate flower development [57]. The homolog of MR8G001578.1 (SPL) in Arabidopsis is a negative regulator of inflorescence identity [58]. Those genes may play roles in red bayberry floral development.

Sex-determining genes are often located in the non-recombining region of sex chromosomes. In the red bayberry, our previous study showed seven putative genes in the $59 \mathrm{~Kb}$ female-specific region of the $\mathrm{W}$ chromosome [7]. We found that all the female-specific genes in F0 (before sexual differentiation) and F1 (flower initiation) displayed low expression with the FPKM value < 5 (Table 1). Hermaphrodite 35S-MrFT2-transformed Arabidopsis plants exhibited a slight reduction in the numbers of stamens (data not shown). Based on the above results, we speculate that ethylene-related gene MR0TCONS_00017483.1 (ACO) may be downstream of the sex-determining gene, and it may interact with the transcription factor MR8G027384.1 (COL4) to control sex differentiation in the red bayberry. This hypothesis needs to be verified in future work and can be tested with external ethylene application in an attempt to alter sex. This could provide effective technical support for cross-breeding between varieties.

Supplementary Materials: The following supporting information can be downloaded at https:// Www.mdpi.com/article/10.3390/horticulturae8020183/s1, Figure S1. Principal component analysis (PCA) of red bayberry floral buds; Figure S2. Venn diagram of the 8889 DEGs in the pair-wise analyses of F1 vs. F0, M1 vs. M0, M0 vs. F0, and M1 vs. F1; Figure S3. KEGG pathway enrichment analysis of the DEGs in M0 vs. F0 (a), M1 vs. F1 (b) and all the 7029 DEGs in the pair-wise M0 vs. F0, and M1 vs. F1 comparisons (c); Figure S4. Distribution of the 415 transcription factors genes in 7029 DEGs; Figure S5. Validation of the expression patterns of ten DEGs via qRT-PCR. Capital letters above the 
black bars represent significant differences between the samples, and values are mean $\pm S E(n=3)$; Table S1. Raw data of sequencing sample in the study; Table S2. Level 2 gene ontology annotation of highly expressed genes in four floral buds; Table S3. KEGG enrichment analysis of highly expressed genes in four floral buds; Table S4. Level 2 gene ontology annotation of middlingly expressed genes in four floral buds; Table S5. KEGG enrichment analysis of middlingly expressed genes in four floral buds; Table S6. Level 2 gene ontology annotation of the DEGs between M0 vs. F0, M1 vs. F1, and 7029 DEGs of the two compared groups in ontologies; Table S7. List of DEGs enriched in plant hormone synthesis and the signal transduction pathway; Table S8. List of hormone-related genes identified in the 7029 DEGs; Table S9. List of hormone-related genes specifically expressed in males and females; Table S10. List of transcription factors identified in the 7029 DEGs; Table S11. List of transcription factor genes specifically expressed in males and females; Table S12. The element enriched in promoter region of hormone-related genes; Table S13. List of genes in the light-green and dark-cyan networks; Table S14. Annotation of genes in light-green module; Table S15. Annotation of genes in dark-cyan module; Table S16. List of primers used for qRT-PCR.

Author Contributions: H.J. (Huimin Jia) and Z.G. designed this study and supervised the work. H.J. (Huimin Jia) performed the bioinformatic analyses and wrote the manuscript. L.Z., Y.W., H.W., H.Z. and H.J. (Huijuan Jia) performed the bioinformatic analyses. H.J. (Huijuan Jia), Y.Z., Y.J., G.W., C.Z. and C.H. collected the samples and generated the raw sequence data. All authors have read and agreed to the published version of the manuscript.

Funding: HM Jia was supported by the National Natural Science Foundation for Young Scientists of China (No. 31901985) and ZS Gao acknowledges support from the National Natural Science Foundation of China (No. 31972364).

Institutional Review Board Statement: Not applicable.

Informed Consent Statement: Not applicable.

Data Availability Statement: The sequencing data have been deposited in the NCBI Sequence Read Archive (http:/ / www.ncbi.nlm.nih.gov/sra/, accessed on 15 February 2022), with the accession number SAMN18515266 and SAMN18515266 for female and male plants, respectively.

Conflicts of Interest: The authors declare no conflict of interest.

\section{References}

1. Renner, S.S. The relative and absolute frequencies of angiosperm sexual systems: Dioecy, monoecy, gynodioecy, and an updated online database. Am. J. Bot. 2014, 101, 1588-1596. [CrossRef]

2. Mitchell, C.H.; Diggle, P.K. The evolution of unisexual flowers: Morphological and functional convergence results from diverse developmental transitions. Am. J. Bot. 2005, 92, 1068-1076. [CrossRef] [PubMed]

3. Diggle, P.K.; Di Stilio, V.S.; Gschwend, A.R.; Golenberg, E.M.; Moore, R.C.; Russell, J.R.; Sinclair, J.P. Multiple developmental processes underlie sex differentiation in angiosperms. Trends Genet. TIG 2011, 27, 368-376. [CrossRef] [PubMed]

4. Chuck, G. Molecular mechanisms of sex determination in monoecious and dioecious plants. Adv. Bot. Res. 2010, 54, 53-83. [CrossRef]

5. Akagi, T.; Henry, I.M.; Tao, R.; Comai, L. A Y-chromosome-encoded small RNA acts as a sex determinant in persimmons. Science 2014, 346, 646-650. [CrossRef]

6. Wang, J.; Na, J.-K.; Yu, Q.; Gschwend, A.R.; Han, J.; Zeng, F.; Aryal, R.; VanBuren, R.; Murray, J.E.; Zhang, W.; et al. Sequencing papaya $X$ and $Y^{h}$ chromosomes reveals molecular basis of incipient sex chromosome evolution. Proc. Natl. Acad. Sci. USA 2012, 109, 13710-13715. [CrossRef]

7. Jia, H.M.; Jia, H.J.; Cai, Q.L.; Wang, Y.; Zhao, H.B.; Yang, W.F.; Wang, G.Y.; Li, Y.H.; Zhan, D.L.; Shen, Y.T.; et al. The red bayberry genome and genetic basis of sex determination. Plant Biotechnol. J. 2019, 17, 397-409. [CrossRef]

8. Tennessen, J.A.; Govindarajulu, R.; Liston, A.; Ashman, T.-L. Homomorphic ZW chromosomes in a wild strawberry show distinctive recombination heterogeneity but a small sex-determining region. New Phytol. 2016, 211, 1412-1423. [CrossRef]

9. Harkess, A.; Zhou, J.; Xu, C.; Bowers, J.E.; Van der Hulst, R.; Ayyampalayam, S.; Mercati, F.; Riccardi, P.; McKain, M.R.; Kakrana, A.; et al. The asparagus genome sheds light on the origin and evolution of a young Y chromosome. Nat. Commun. 2017, 8, 1279. [CrossRef]

10. Harkess, A.; Huang, K.; van der Hulst, R.; Tissen, B.; Caplan, J.L.; Koppula, A.; Batish, M.; Meyers, B.C.; Leebens-Mack, J. Sex Determination by Two Y-Linked Genes in Garden Asparagus. Plant Cell 2020, 32, 1790-1796. [CrossRef]

11. Akagi, T.; Henry, I.M.; Ohtani, H.; Morimoto, T.; Beppu, K.; Kataoka, I.; Tao, R. A Y-Encoded Suppressor of Feminization Arose via Lineage-Specific Duplication of a Cytokinin Response Regulator in Kiwifruit. Plant Cell 2018, 30, 780-795. [CrossRef] [PubMed] 
12. Akagi, T.; Pilkington, S.M.; Varkonyi-Gasic, E.; Henry, I.M.; Sugano, S.S.; Sonoda, M.; Firl, A.; McNeilage, M.A.; Douglas, M.J.; Wang, T.; et al. Two Y-chromosome-encoded genes determine sex in kiwifruit. Nat. Plants 2019, 5, 801-809. [CrossRef]

13. Aryal, R.; Ming, R. Sex determination in flowering plants: Papaya as a model system. Plant Sci. 2014, 217-218, 56-62. [CrossRef] [PubMed]

14. Heikrujam, M.; Sharma, K.; Prasad, M.; Agrawal, V. Review on different mechanisms of sex determination and sex-linked molecular markers in dioecious crops: A current update. Euphytica 2015, 201, 161-194. [CrossRef]

15. Zhang, H.; Li, S.; Yang, L.; Cai, G.; Chen, H.; Gao, D.; Lin, T.; Cui, Q.; Wang, D.; Li, Z.; et al. Gain-of-function of the 1-aminocyclopropane-1-carboxylate synthase gene ACS1G induces female flower development in cucumber gynoecy. Plant Cell 2021, 33, 306-321. [CrossRef] [PubMed]

16. Li, Z.; Huang, S.; Liu, S.; Pan, J.; Zhang, Z.; Tao, Q.; Shi, Q.; Jia, Z.; Zhang, W.; Chen, H.; et al. Molecular Isolation of the M Gene Suggests That a Conserved-Residue Conversion Induces the Formation of Bisexual Flowers in Cucumber Plants. Genetics 2009, 182, 1381-1385. [CrossRef] [PubMed]

17. Boualem, A.; Troadec, C.; Camps, C.; Lemhemdi, A.; Morin, H.; Sari, M.-A.; Fraenkel-Zagouri, R.; Kovalski, I.; Dogimont, C.; Perl-Treves, R.; et al. A cucurbit androecy gene reveals how unisexual flowers develop and dioecy emerges. Science 2015, 350, 688-691. [CrossRef]

18. Chen, H.; Sun, J.; Li, S.; Cui, Q.; Zhang, H.; Xin, F.; Wang, H.; Lin, T.; Gao, D.; Wang, S.; et al. An ACC Oxidase Gene Essential for Cucumber Carpel Development. Mol. Plant 2016, 9, 1315-1327. [CrossRef]

19. Huguet, V.; Gouy, M.; Normand, P.; Zimpfer, J.F.; Fernandez, M.P. Molecular phylogeny of Myricaceae: A reexamination of host-symbiont specificity. Mol. Phylogenet. Evol. 2005, 34, 557-568. [CrossRef]

20. Chen, K.S.; Xu, C.J.; Zhang, B.; Ferguson, I.B. Red bayberry: Botany and horticulture. Hortic. Rev. 2004, 30, 83-114. [CrossRef]

21. Wilbur, R.L. The Myricaceae of the United States and Canada: Genera, subgenera, and series. SIDA Contrib. Bot. 1994, $16,93-107$.

22. Jia, H.M.; Jiao, Y.; Wang, G.Y.; Li, Y.H.; Jia, H.J.; Wu, H.X.; Chai, C.Y.; Dong, X.; Guo, Y.P.; Zhang, L.P.; et al. Genetic diversity of male and female Chinese bayberry (Myrica rubra) populations and identification of sex-associated markers. BMC Genom. 2015, 16, 394. [CrossRef] [PubMed]

23. Wang, Y.; Jia, H.-M.; Shen, Y.-T.; Zhao, H.-B.; Yang, Q.-S.; Zhu, C.-Q.; Sun, D.-L.; Wang, G.-Y.; Zhou, C.-C.; Jiao, Y.; et al. Construction of an anchoring SSR marker genetic linkage map and detection of a sex-linked region in two dioecious populations of red bayberry. Hortic. Res. 2020, 7, 53. [CrossRef] [PubMed]

24. Liu, J.; Chen, L.-Y.; Zhou, P.; Liao, Z.; Lin, H.; Yu, Q.; Ming, R. Sex biased expression of hormone related genes at early stage of sex differentiation in papaya flowers. Hortic. Res. 2021, 8, 147. [CrossRef] [PubMed]

25. Liu, Z.; Wang, H.; Xu, Z.; Zhang, H.; Li, G.; Wang, X.; Qian, W. Transcriptome profiling of differentially expressed genes of male and female inflorescences in spinach (Spinacia oleracea L.). Genome 2021, 64, 777-788. [CrossRef] [PubMed]

26. Li, N.; Meng, Z.; Tao, M.; Wang, Y.; Zhang, Y.; Li, S.; Gao, W.; Deng, C. Comparative transcriptome analysis of male and female flowers in Spinacia oleracea L. BMC Genom. 2020, 21, 850. [CrossRef]

27. Hui, W.; Yang, Y.; Wu, G.; Peng, C.; Chen, X.; Zayed, M.Z. Transcriptome profile analysis reveals the regulation mechanism of floral sex differentiation in Jatropha curcas L. Sci. Rep. 2017, 7, 16421. [CrossRef]

28. Zhang, Z.; Lin, Q.; Zhong, Q. Monoecious Mutant Reveals New Insights into Male and Female Inflorescence Development in the Chinese Bayberry (Morella rubra). HortSci. Horts 2017, 52, 343. [CrossRef]

29. Ao, C.Q. Developmental origins of the conjoined twin mature embryo sacs in Smilax davidiana, with special notes on the formation of their embryos and endosperms. Am. J. Bot. 2013, 100, 2509-2515. [CrossRef]

30. Chen, Y.; Chen, Y.; Shi, C.; Huang, Z.; Zhang, Y.; Li, S.; Li, Y.; Ye, J.; Yu, C.; Li, Z.; et al. SOAPnuke: A MapReduce accelerationsupported software for integrated quality control and preprocessing of high-throughput sequencing data. GigaScience 2017, 7, 1-6. [CrossRef]

31. Bolger, A.M.; Lohse, M.; Usadel, B. Trimmomatic: A flexible trimmer for Illumina sequence data. Bioinformatics 2014, 30, 2114-2120 [CrossRef] [PubMed]

32. Kim, D.; Langmead, B.; Salzberg, S.L. HISAT: A fast spliced aligner with low memory requirements. Nat. Methods 2015, 12, 357-360. [CrossRef] [PubMed]

33. Langmead, B.; Salzberg, S.L. Fast gapped-read alignment with Bowtie 2. Nat. Methods 2012, 9, 357-359. [CrossRef] [PubMed]

34. Li, B.; Dewey, C.N. RSEM: Accurate transcript quantification from RNA-Seq data with or without a reference genome. BMC Bioinform. 2011, 12, 323. [CrossRef]

35. Conesa, A.; Gotz, S.; Garcia-Gomez, J.M.; Terol, J.; Talon, M.; Robles, M. Blast2GO: A universal tool for annotation, visualization and analysis in functional genomics research. Bioinformatics 2005, 21, 3674-3676. [CrossRef]

36. Wang, L.; Feng, Z.; Wang, X.; Wang, X.; Zhang, X. DEGseq: An R package for identifying differentially expressed genes from RNA-seq data. Bioinformatics 2010, 26, 136-138. [CrossRef]

37. Chen, C.; Chen, H.; Zhang, Y.; Thomas, H.R.; Frank, M.H.; He, Y.; Xia, R. TBtools: An Integrative Toolkit Developed for Interactive Analyses of Big Biological Data. Mol. Plant 2020,13, 1194-1202. [CrossRef]

38. Langfelder, P.; Horvath, S. WGCNA: An R package for weighted correlation network analysis. BMC Bioinform. 2008, 9, 559. [CrossRef] 
39. Ni, J.; Bai, S.; Zhao, Y.; Qian, M.; Tao, R.; Yin, L.; Gao, L.; Teng, Y. Ethylene response factors Pp4ERF24 and Pp12ERF96 regulate blue light-induced anthocyanin biosynthesis in 'Red Zaosu' pear fruits by interacting with MYB114. Plant Mol. Biol. 2019, 99, 67-78. [CrossRef]

40. Livak, K.J.; Schmittgen, T.D. Analysis of relative gene expression data using real-time quantitative PCR and the 2(-Delta Delta C(T)) Method. Methods 2001, 25, 402-408. [CrossRef]

41. Khryanin, V.N. Role of Phytohormones in Sex Differentiation in Plants. Russ. J. Plant Physiol. 2002, 49, 545-551. [CrossRef]

42. Yamasaki, S.; Fujii, N.; Matsuura, S.; Mizusawa, H.; Takahashi, H. The M Locus and Ethylene-Controlled Sex Determination in Andromonoecious Cucumber Plants. Plant Cell Physiol. 2001, 42, 608-619. [CrossRef] [PubMed]

43. Tao, Q.; Niu, H.; Wang, Z.; Zhang, W.; Wang, H.; Wang, S.; Zhang, X.; Li, Z. Ethylene responsive factor ERF110 mediates ethylene-regulated transcription of a sex determination-related orthologous gene in two Cucumis species. J. Exp. Bot. 2018, 69, 2953-2965. [CrossRef] [PubMed]

44. Bensen, R.J.; Johal, G.S.; Crane, V.C.; Tossberg, J.T.; Schnable, P.S.; Meeley, R.B.; Briggs, S.P. Cloning and characterization of the maize An1 gene. Plant Cell 1995, 7, 75-84. [CrossRef] [PubMed]

45. Hartwig, T.; Chuck, G.S.; Fujioka, S.; Klempien, A.; Weizbauer, R.; Potluri, D.P.; Choe, S.; Johal, G.S.; Schulz, B. Brassinosteroid control of sex determination in maize. Proc. Natl. Acad. Sci. USA 2011, 108, 19814-19819. [CrossRef] [PubMed]

46. Achard, P.; Baghour, M.; Chapple, A.; Hedden, P.; Van Der Straeten, D.; Genschik, P.; Moritz, T.; Harberd, N.P. The plant stress hormone ethylene controls floral transition via DELLA-dependent regulation of floral meristem-identity genes. Proc. Natl. Acad. Sci. USA 2007, 104, 6484-6489. [CrossRef] [PubMed]

47. Martinez, C.; Pons, E.; Prats, G.; Leon, J. Salicylic acid regulates flowering time and links defence responses and reproductive development. Plant J. 2004, 37, 209-217. [CrossRef]

48. Jin, J.B.; Jin, Y.H.; Lee, J.; Miura, K.; Yoo, C.Y.; Kim, W.-Y.; Van Oosten, M.; Hyun, Y.; Somers, D.E.; Lee, I.; et al. The SUMO E3 ligase, AtS1Z1, regulates flowering by controlling a salicylic acid-mediated floral promotion pathway and through affects on FLC chromatin structure. Plant J. 2008, 53, 530-540. [CrossRef]

49. Van der Meer, I.M.; Stam, M.E.; van Tunen, A.J.; Mol, J.N.; Stuitje, A.R. Antisense inhibition of flavonoid biosynthesis in petunia anthers results in male sterility. Plant Cell 1992, 4, 253-262. [CrossRef]

50. Martin, A.; Troadec, C.; Boualem, A.; Rajab, M.; Fernandez, R.; Morin, H.; Pitrat, M.; Dogimont, C.; Bendahmane, A. A transposon-induced epigenetic change leads to sex determination in melon. Nature 2009, 461, 1135-1138. [CrossRef]

51. Steinbach, Y. The Arabidopsis thaliana CONSTANS-LIKE 4 (COL4)-A Modulator of Flowering Time. Front. Plant Sci. 2019, 10, 651. [CrossRef] [PubMed]

52. Wu, W.; Zheng, X.M.; Chen, D.; Zhang, Y.; Ma, W.; Zhang, H.; Sun, L.; Yang, Z.; Zhao, C.; Zhan, X.; et al. OsCOL16, encoding a CONSTANS-like protein, represses flowering by up-regulating Ghd7 expression in rice. Plant Sci. Int. J. Exp. Plant Biol. 2017, 260, 60-69. [CrossRef] [PubMed]

53. Lee, J.; Lee, I. Regulation and function of SOC1, a flowering pathway integrator. J. Exp. Bot. 2010, 61, 2247-2254. [CrossRef] [PubMed]

54. Moon, J.; Suh, S.S.; Lee, H.; Choi, K.R.; Hong, C.B.; Paek, N.C.; Kim, S.G.; Lee, I. The SOC1 MADS-box gene integrates vernalization and gibberellin signals for flowering in Arabidopsis. Plant. J. Cell Mol. Biol. 2003, 35, 613-623. [CrossRef] [PubMed]

55. García, A.; Aguado, E.; Martínez, C.; Loska, D.; Beltrán, S.; Valenzuela, J.L.; Garrido, D.; Jamilena, M. The ethylene receptors CPETR1A and CPETR2B cooperate in the control of sex determination in Cucurbita pepo. J. Exp. Bot. 2020, 71, 154-167. [CrossRef] [PubMed]

56. Wang, B.; Jin, S.-H.; Hu, H.-Q.; Sun, Y.-G.; Wang, Y.-W.; Han, P.; Hou, B.-K. UGT87A2, an Arabidopsis glycosyltransferase, regulates flowering time via FLOWERING LOCUS C. New Phytol. 2012, 194, 666-675. [CrossRef]

57. Levy, Y.Y.; Mesnage, S.; Mylne, J.S.; Gendall, A.R.; Dean, C. Multiple Roles of Arabidopsis VRN1 in Vernalization and Flowering Time Control. Science 2002, 297, 243-246. [CrossRef]

58. Stone, J.M.; Liang, X.; Nekl, E.R.; Stiers, J.J. Arabidopsis AtSPL14, a plant-specific SBP-domain transcription factor, participates in plant development and sensitivity to fumonisin B1. Plant J. 2005, 41, 744-754. [CrossRef] 